\title{
On Offset Boosting in Chaotic System
}

\author{
Chunbiao Li ${ }^{(\mathbb{D} *, t, 1}$, Yicheng Jiang ${ }^{(\mathbb{D}) *,+, 2}$ and Xu Ma ${ }^{(\mathbb{D} *,+, 3}$ \\ ${ }^{*}$ School of Artificial Intelligence, Nanjing University of Information Science and Technology, Nanjing 210044, China, ${ }^{\dagger}$ Jiangsu Collaborative Innovation Center \\ of Atmospheric Environment and Equipment Technology (CICAEET), Nanjing University of Information Science and Technology, Nanjing 210044 , China.
}

\begin{abstract}
Offset boosting is an important issue for chaos control due to its broadband property and polarity control. There are two main approaches to realize offset boosting. One is resort to parameter introducing where an offset booster realizes attractor boosting. The other one is by the means of periodic function or absolute value function where any self-reproduced or doubled attractors with diverse offset are extracted out by a specific initial condition. The former also provides a unique window for observing multistability and the latter gives the direction for constructing desired multistability.
\end{abstract}

KEYWORDS

Offset boosting

Chaos control

Multistability

\section{INTRODUCTION}

Chaotic signal is wide used in chaos-based information engineering including chaotic secure communication, image encryption and neural signal processing. Any chaotic signal has its inherent features namely identified as scale (C. Sprott and Xiong 2015; C. Sprott 2010; Gu et al. 2021; Lu et al. 2019; Liu et al. 2020; Wang et al. 2020; Zhao et al. 2020; Akgul et al. 2016, 2019; Falco et al. 2012) and offset (Li et al. 2019, 2017a; Liu et al. 2020; Li and Sprott 2017; Li et al. 2021, 2017b; Kingni et al. 2020; Ma et al. 2021; Zhang et al. 2018; Mezatio et al. 2019; Bao et al. 2020; Chen et al. 2020; Zhang et al. 2020; Wu et al. 2019a; Ding et al. 2020). For rescaling a chaotic signal, people usually design dynamical systems with amplitude control from the very beginning. In fact, for an attractor in phase space, amplitude control typically gets involved with offset boosting. Offset boosting means that the attractor is moved in phase space in any direction, which indicates that the average value of corresponding variable is rescaled accordingly. In a differential equation, a simple substation of $x_{i} \rightarrow x_{i}+c$ revises the average value of $x_{i}$ without revising the left hand of its master system. Therefore it looks very simple in the mathematic view. However, offset boosting is such an important issue in chaotic system since that it gives a direct way for an engineer to transform a bipolar chaotic signal to a unipolar one. And besides this, it seems that offset boosting shows more varieties than our imagination such as attractor boosting, attractor self-reproducing (Li et al. 2017a), attractor doubling (Li et al. 2019), conditional symmetry (Li et al. 2020c),

\footnotetext{
Manuscript received: 1 July 2021 ,

Revised: 28 July 2021 ,

Accepted: 2 August 2021

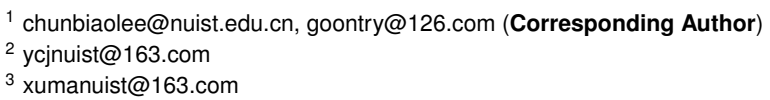

time-reversible symmetry (Li and Sprott 2017) or even repellor construction (Li et al. 2021). For this reason, offset boosting has attracted great interests recently both in continuous system and in discrete maps. Researchers pays great effort to learn how to get those attractors controlled by parameters (Li et al. 2017b; Kingni et al. 2020; Ma et al. 2021; Zhang et al. 2018; Mezatio et al. 2019) or by initial conditions (Bao et al. 2020; Chen et al. 2020; Zhang et al. 2020; Wu et al. 2019a; Ding et al. 2020). Even in those memristive systems (Chen et al. 2019; Kengne et al. 2018; Lu et al. 2020; Wu et al. 2019b; Yuan et al. 2019) offset boosting is still a hot spot for discussion.

As shown in Fig. 1, offset booster can be attached in a chaotic system for attractor boosting, which means that the newly derived attractor stays at different positions controlled by the offset constant. Typically, to realize offset boosting a unified constant is necessary to insert into multiple terms if the corresponding variable appears many times. Specific variable-boostable chaotic systems (Li and Sprott 2016) give the simple possibility for offset boosting since in the right hand there is a variable appearing only once. However, for attractor self-reproducing, periodic functions including multiple similar linear segments are needed where any specific initial condition can visit its most closed attractor obeying the distribution of basin of attraction. In this work, parameter-oriented offset boosting and initial-condition-oriented are systematically discussed based on system VB14 (Li and Sprott 2016), as indicated in Table.1. The case of absolute value function introducing is not listed in the table for easy discussion in the following text where more parameters are embedded and correspondingly the original system is changed more dramatically. In section 2, we discuss how to insert a constant to realize offset boosting in some dimensions of a system. In section 3, periodic functions specifically trigonometric functions are addressed for initial condition-triggered offset boosting. In section 4 , absolute value functions are introduced for 


$\begin{array}{lll}\text { Cases } & \begin{array}{l}\text { Constant or function introducing for offset } \\ \text { boosting }\end{array} & \text { Research focus } \\ \text { A } & \mathrm{F}(x)=x+d, \mathrm{G}(y)=y & \text { Attractor Boosting } \\ \text { B } & \mathrm{F}(x)=x, \mathrm{G}(y)=y+h & \\ \text { C } & \mathrm{F}(x)=3 \sin (x), \mathrm{G}(y)=y & \text { Attractor Self-reproducing and Attractor Growing } \\ \text { D } & \mathrm{F}(x)=1.8 \sin (1.3 x), \mathrm{G}(y)=y & \\ \text { E } & \mathrm{F}(x)=x, \mathrm{G}(y)=2 \cos (y) \\ \mathrm{F} & \mathrm{F}(x)=3 \tan (0.5 x), \mathrm{G}(y)=\sin (y) \\ \mathrm{G} & \mathrm{F}(x)=x+d, \mathrm{G}(y)=\sin (y) & \end{array}$

attractor doubling. In section 6, absolute value function is applied for polarity reverse for the observation of conditional symmetry and repellor construction. Conclusions are wrapped in the last section.

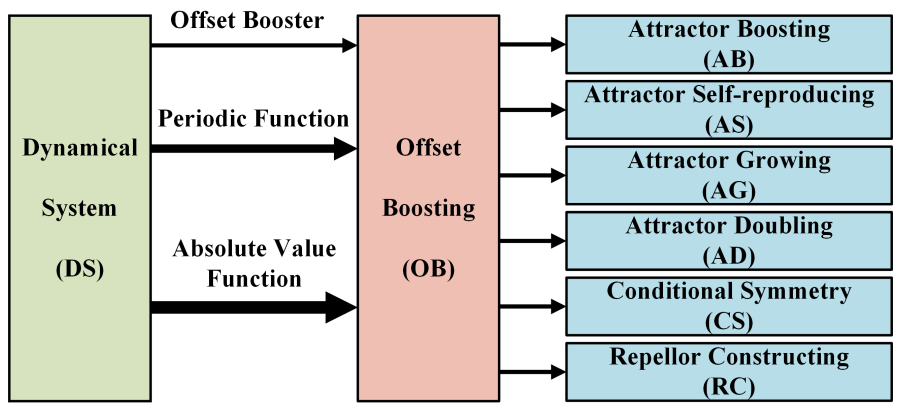

Figure 1 Offset boosting in a dynamical system.

\section{OFFSET BOOSTING BY INTRODUCING CONSTANT TERMS}

As mentioned above, to realize offset boosting in a dynamical system, a direct method of introducing a constant in any of the dimension can be applied for this target. For example, we introduce a constant in the variable $\mathrm{x}$ in VB14, correspondingly system (1) turns to be system (2) where only an extra single constant appear in the right hand,

$$
\begin{gathered}
\left\{\begin{array}{l}
\dot{x}=1-a y z \\
\dot{y}=z^{2}-z \\
\dot{z}=x-b z
\end{array}\right. \\
\left\{\begin{array}{l}
\dot{x}=1-a y z \\
\dot{y}=z^{2}-z \\
\dot{z}=x+d-b z
\end{array}\right.
\end{gathered}
$$

Correspondingly, the original attractor, shown in Fig. 2, will be shifted in the $x$ dimension, shown in Fig. 3, without shaking the Lyapunov exponents but spitting out the chaotic signal $x$ in a smoothly revised average, shown in Fig. 4.
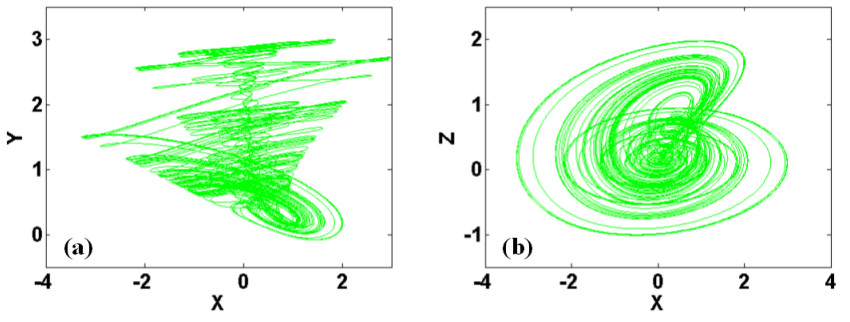

Figure 2 Chaotic attractor of system (1) with $a=3.55, b=0.5$ under initial condition $(1,0,1)$ : (a) $x-y$, (b) $x-z$.
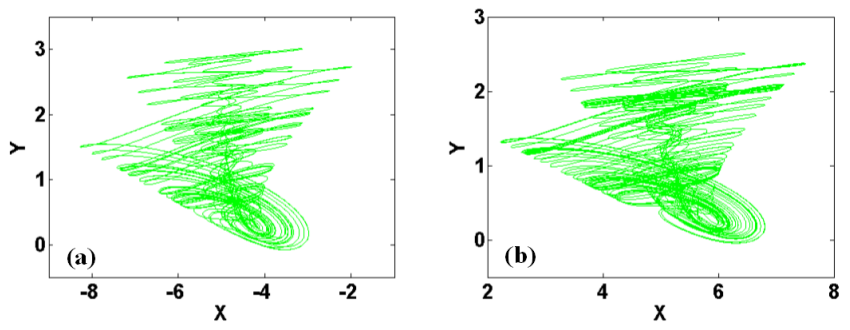

Figure 3 Shifted chaotic attractors in system (2) with $a=3.55$, $b=0.5$ under initial condition $(1-d, 0,1)$ (Case A in Table 1): (a) $d=5,(\mathrm{~b}) d=-5$.
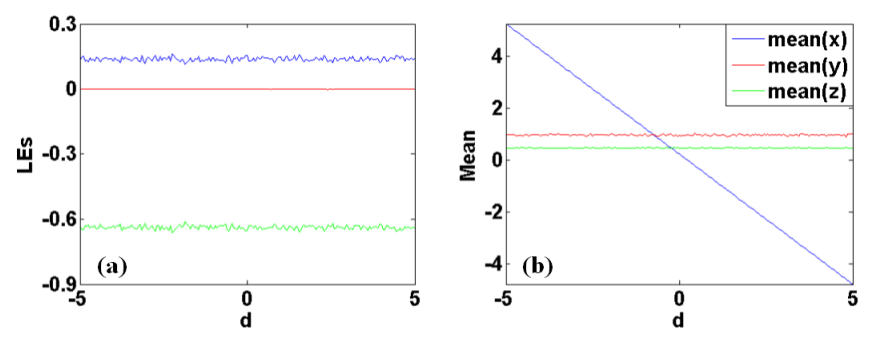

Figure 4 Offset boosting of $\mathrm{x}$ in system (2) with $a=3.55, b=0.5$ under initial condition $(1-d, 0,1)$ : (a) Lyapunov exponents, (b) average values. 
The operation for offset boosting can be repeated in other dimensions. But this does not mean that all this kind of operations share the same complexity for a dynamical system and correspondingly does not benefit circuit realization equally. For example, it is shown that even an exactly similar operation of $y \rightarrow y+h$ needs much more effort for circuit realization as indicated in system (3). For system (2), a newly introduced direct current source revise the average value of $x$, while for system (3) (Case B in Table. 1), a new feedback of $-a h z$ should be attached in the $x$ dimension. The offset of $y$ is based on the adjustable resistance in the branch of $x$-dimension, which is not getting worse. In fact, the constant $h$ still returns the average-value-revised $y$ with unified Lyapunov exponents, as shown in Fig. 5. In some circumstances, it will be much more catastrophic even a single constant is needed for a variable but multiple existence of this variable brings much more complexity.

$$
\left\{\begin{array}{l}
\dot{x}=1-a(y+h) z \\
\dot{y}=z^{2}-z \\
\dot{z}=x-b z
\end{array}\right.
$$
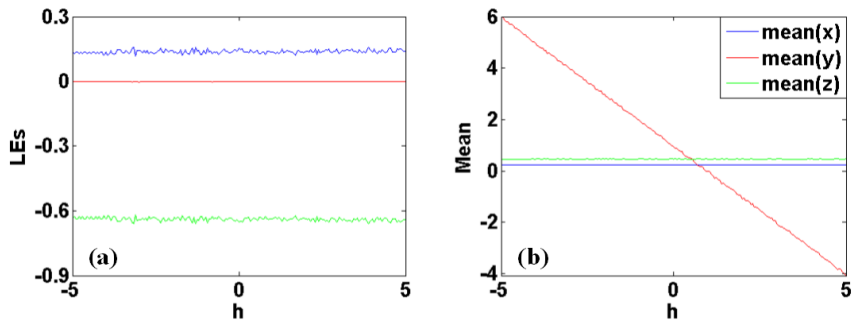

Figure 5 Offset boosting of y in system (3) with $a=3.55$, $b=0.5$ under initial condition $(1,-h, 1)$ (Case B in Table 1): (a) Lyapunov exponents, (b) average values.

\section{OFFSET BOOSTING BY INTRODUCING PERIODIC FUNC- TIONS}

Offset boosting can be realized in a hidden mode where a periodic function is applied for attractor boosting. In this direction, as pointed in ( $\mathrm{Li}$ et al. 2017a), periodic trigonometric function is introduced for hidden offset boosting by initial condition. In this example, a sinusoidal is equipped as,

$$
\left\{\begin{array}{l}
\dot{x}=1-a \mathrm{G}(y) z \\
\dot{y}=z^{2}-z \\
\dot{z}=F(x)-b z
\end{array}\right.
$$

where $\mathrm{G}(y)=y, \mathrm{~F}(x)=3 \sin (x)$. Thus, system (4) (Case C in Table 1 ) is a self-reproducing system giving infinitely many coexisting attractors, which can be extracted by various initial conditions, as shown in Fig. 6, eight coexisting attractors are given, each of which is of the same shape with same Lyapunov exponents $(0.23401,0$, $-0.73402)$ as shown in Fig. 7. The stout structure of attractor in Fig. 6 is flattened for the increasing scale in the $x$-axis. In fact, system (4) reproduces infinitely many attractors standing on different positions in the $x$-axis but with unified Lyapunov exponents, as shown in Fig. 8. Note that the step-growing average value shows the offset boosting triggered by initial conditions. Each step represents a corresponding attractor and the whole sinusoidal-like evolution reveals the trigonometric nonlinearity.
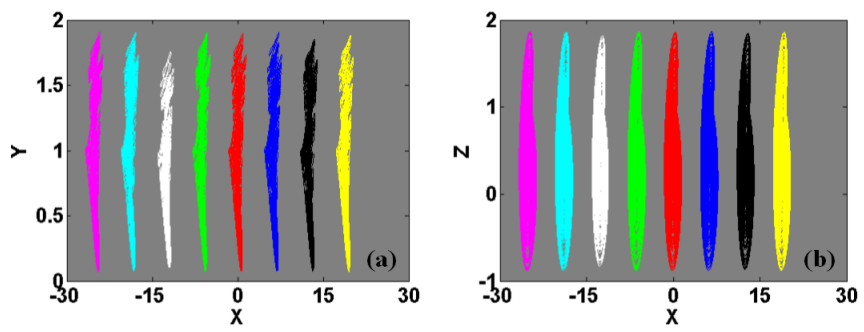

Figure 6 Coexisting attractors in system (4) with $\mathrm{G}(y)=y, \mathrm{~F}(x)$ $=3 \sin (x), a=3.55, b=0.5$ (Case C in Table. 1): (a) $x-y$, (b) $x-z$. Each attractor is under different initial conditions (IC). Here cyan is for IC $=(-5,0,1)$, pink is for IC $=(-4,0,1)$, yellow is for IC $=$ $(-3,0,1)$, red is for $(-2,0,1)$, green for IC $=(1,0,1)$, blue is for IC $=(5,0,1)$, white is for $\mathrm{IC}=(8,0,1)$, black is for $(11,0,1)$.
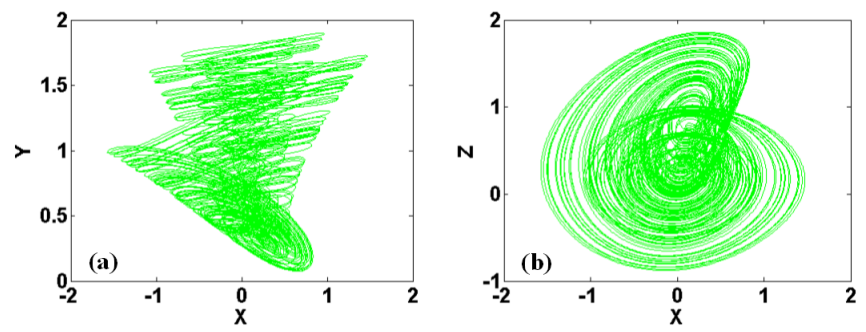

Figure 7 Chaotic attractor of system (4) with $\mathrm{G}(y)=y, \mathrm{~F}(x)=$ $3 \sin (x), a=3.55, b=0.5$ in principal interval when initial condition $\mathrm{IC}=(-2,0,1)$ : (a) $x-y$, (b) $x-z$.
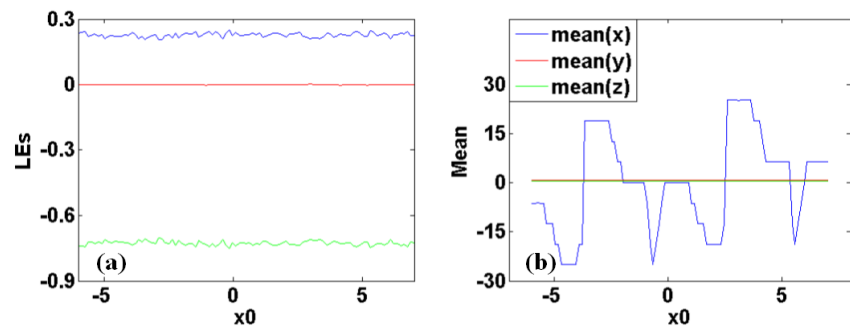

Figure 8 Offset boosting of system (4) with $\mathrm{G}(y)=y, \mathrm{~F}(x)=$ $3 \sin (x), a=3.55, b=0.5$, and IC $=\left(x_{0}, 0,1\right), x_{0}$ varies in $[-6$, 7]: (a) Lyapunov exponents, (b) average values.
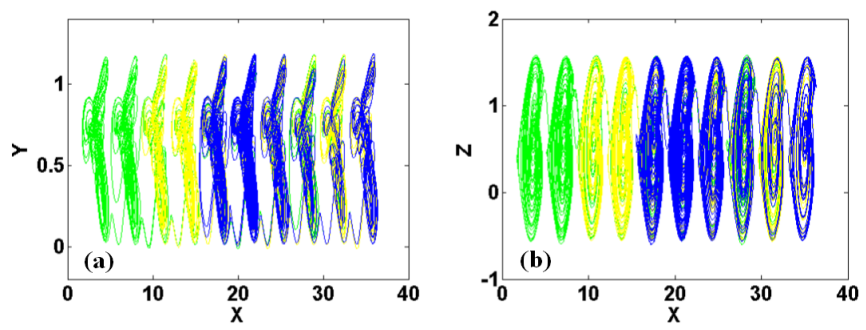

Figure 9 Attractor growing in system (4) with $\mathrm{G}(y)=y, \mathrm{~F}(x)=$ $1.2 \sin (1.8 x), a=3.6, b=0.5$ and time duration $T=1000$. Here green is for IC $=(1,0,1)$, yellow is for IC $=(5,0,1)$, blue is for IC $=(10,0,1):$ (a) $x-y$, (b) $x-z$. 
Meanwhile revising the feedback of sinusoidal function, attractor growing appears as predicted. In this case the introduced functions in system (4) are: $\mathrm{G}(y)=y, \mathrm{~F}(x)=1.2 \sin (1.8 x)$ (Case D in Table. 1). Different initial values select the start point for attractor growing indicated in green, yellow and blue in Fig. 9. This is the typical phenomenon of homogenous multistability. Unified Lyapunov exponents are proved along with randomly increasing of the average value of $x$ as shown in Fig.10.
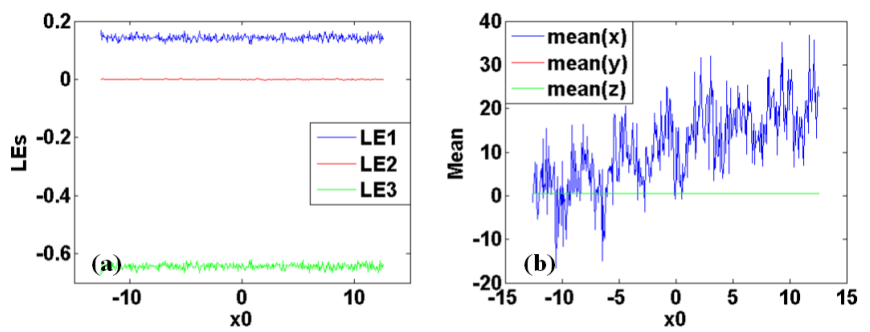

Figure 10 Feature of the growing attractor in system (4) with $\mathrm{G}(y)=y, \mathrm{~F}(x)=1.2 \sin (1.8 x), a=3.6, b=0.5$ and time duration of $T=1000$ under the initial condition $\left[x_{0}, 0,1\right]$, where $x_{0}$ varies in $[-4 \pi, 4 \pi]$ : (a) Lyapunov exponents, (b) average variables.

The initial-condition-oriented offset boosting can extend to other dimension with the same approach. Here a cosine function is introduced in the y dimension in system (4) by $G(y)=2 \cos (y)$, $\mathrm{F}(x)=x($ Case $\mathrm{E}$ in Table. 1$)$,

$$
\left\{\begin{array}{l}
\dot{x}=1-2 a \cos (y) z \\
\dot{y}=z^{2}-z \\
\dot{z}=x-b z
\end{array}\right.
$$

As shown in Fig. 11, when $a=2.2, b=0.7$ and initial condition IC $=(1,0,1)$, system $(5)$ exhibits a chaotic attractor with Lyapunov exponents $(0.13415,0,-0.83416)$. For the same reason of periodicity, system (5) is a self-reproducing system giving infinitely many coexisting attractors with different average values in the $y$-dimension, as shown in Fig. 12. Almost unchanged Lyapunov exponents can be seen in Fig. 13, where linearly modulated offset in the y-dimension shows up. There is no conflict in Fig. 8 and Fig. 13. In Fig. 8, the initial condition of $x_{0}$ varies in a continuous way in region of $[-6,7]$, the sinusoidal-like evolution reveals the trigonometric nonlinearity combined with the fractal structure of basin of attraction. Meanwhile in Fig. 13, the initial condition of $y_{0}$ varies in a discrete way in the period of cosine function $2 n \pi$, the linearly rescaled offset also betrays that the basins for each attractor stand apart from each other in general.

All the operations can be mixed together for flexible offset boosting in any dimension. And furthermore, the attractor selfreproducing can be achieved by other periodic functions. For example, periodic functions are introduced into system (4) in both $x$ and $y$ dimension. Here $\mathrm{G}(y)$ and $\mathrm{F}(x)$ are selected from other trigonometric functions. When $a=3.57, b=0.7, \mathrm{~F}(x)=3 \tan (0.5 x)$, $\mathrm{G}(y)=\sin (y)$ (Case $F$ in Table. 1), infinitely many attractors scatter in the $x$-dimension and $y$-dimension with corresponding attractor space under unified Lyapunov exponents $(0.22152,0,-0.93029)$. In $x$-dimension, the attractor distance is defined by the period of tangent function while in $y$-dimension the attractor distance is defined by the period of sinusoidal function. The attractor distances in $x$-dimension and $y$-dimension are equal this time, which
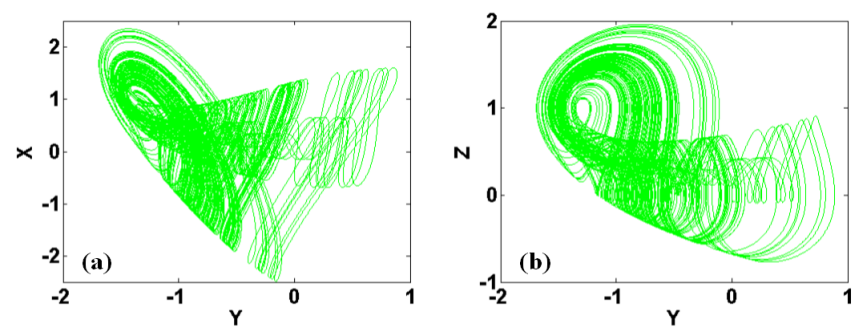

Figure 11 Chaotic attractor of system (5) (case E in Table. 1) with $a=2.2, b=0.7$ under initial condition $(1,0,1)$ : (a) $y-x$, (b) $y-z$.
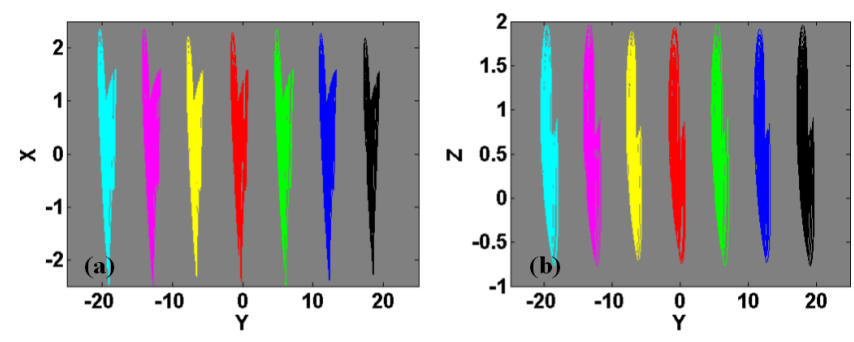

Figure 12 Coexisting attractors in system (5) with $a=2.2, b=$ 0.7 , and IC $=\left(1, y_{0}, 1\right)$ : (a) $y-x$, (b) $y-z$. Here cyan is for $y_{0}=-6 \pi$, pink is for $y_{0}=-4 \pi$, yellow is for $y_{0}=-2 \pi$, red is for $y_{0}=0$, green is for $y_{0}=2 \pi$, blue is for $y_{0}=4 \pi$, black is for $y_{0}=6 \pi$.
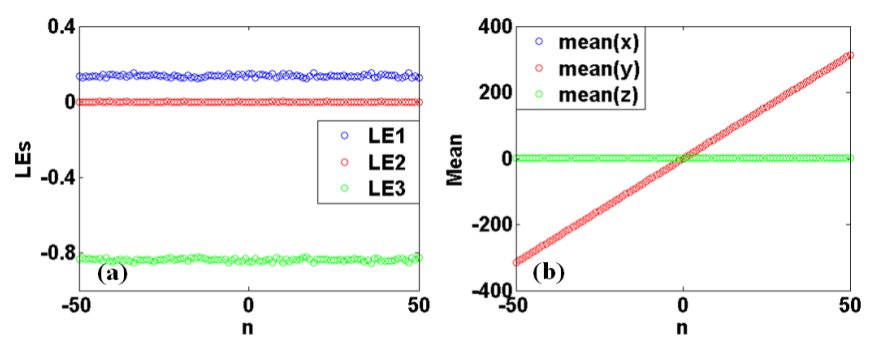

Figure 13 Offset boosting of system (5) with $a=2.2, b=0.7$, and IC $=\left(1, y_{0}, 1\right), y_{0}=2 \mathrm{n} \pi$ : (a) Lyapunov exponents, (b) average values.
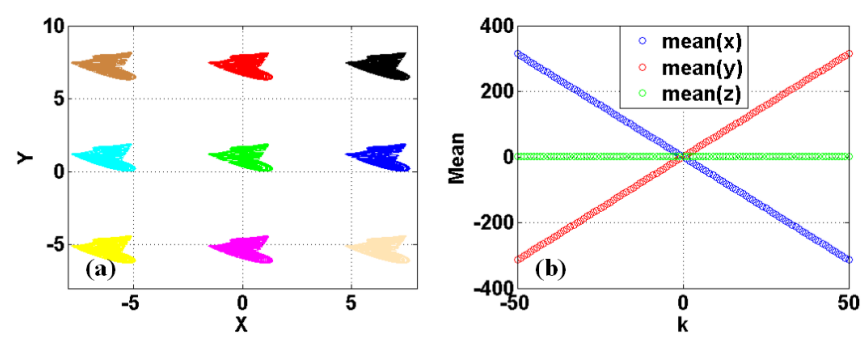

Figure 14 Lattice of strange attractors in system (4) with $a=3.57$, $b=0.7, \mathrm{~F}(x)=3 \tan (0.5 x), \mathrm{G}(y)=\sin (y)$ (Case F in Table. 1): (a) Coexisting strange attractors when initial conditions are $(1+2 k \pi$, $0+2 l \pi, 1(-1 \leq k, l \in Z \leq 1))$, (b) regulated offset when initial conditions are $(1-2 k \pi, 0+2 k \pi, 1(-50 \leq k \in Z \leq 50))$. 
is because both functions of $\tan (0.5 x)$ and $\sin (y)$ have same period of $2 \pi$, as shown in Fig. 14 .

Combined regime of offset boosting can be realized in the following system,

$$
\left\{\begin{array}{l}
\dot{x}=1-a \mathrm{G}(y) z \\
\dot{y}=z^{2}-z \\
\dot{z}=x+d-b z
\end{array}\right.
$$

where the offset boosting in the $x$ dimension is controlled by the constant $d$, while the offset boosting in the $y$ dimension is oriented by the initial condition of $y$ (Case G in Table. 1). When $a=4.5$, $b=0.7, \mathrm{~F}(x)=x+d, \mathrm{G}(y)=\sin (y)$, infinitely many attractors scatter in the $y$-dimension. Meanwhile the location in $x$-axis is set by the constant $d$, as shown in Fig. 15. All these coexisting attractors and constant-controlled attractors share a unified set of Lyapunov exponents (0.12917, 0, -0.82917).
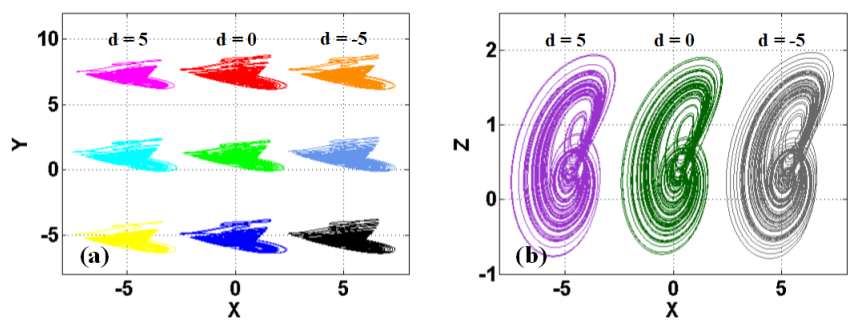

Figure 15 Infinitely many attractors shifted by d in system (6) with $a=4.5, b=0.7, \mathrm{G}(y)=\sin (y)$ (Case G in Table. 1): (a) $x-y$, (b) $x-z$.

\section{ATTRACTOR DOUBLING BY INTRODUCING ABSOLUTE VALUE FUNCTIONS}

Offset boosting from a substitution of absolute value function can bring doubled coexisting attractors (Li et al. 2019). For example, take $y \rightarrow|y|-c$ like,

$$
\left\{\begin{array}{l}
\dot{x}=1-a(|y|-c) z \\
\dot{y}=\operatorname{sgn}(y)\left(z^{2}-\mathrm{z}\right) \\
\dot{z}=x+d-b z
\end{array}\right.
$$

The original attractor obtained its reproducing in the dimension of $y$, pseudo-double-scroll attractor with Lyapunov exponents $(0.1477,0,-0.64335)$ is as shown in Fig. 16. Combined regime of offset boosting can be realized when the offset booster $d$ is not zero. As shown in Fig. 17, the pseudo-double-scroll attractor is controlled locating at various positions in the dimension of $x$ according to the offset booster $d$. Furthermore, the distance between any two doubled coexisting attractors can be controlled by selecting a propitiate value of $c$ in the absolute value function. As plotted in Fig. 18, when $c=1$, doubled coexisting attractors stand separately at both sides in the dimension of $y$. If we hide the obvious independent constant $d$ as the period in the $x$-dimension trigonometric function as,

$$
\left\{\begin{array}{l}
\dot{x}=1-a(|y|-c) z \\
\dot{y}=\operatorname{sgn}(y)\left(z^{2}-\mathrm{z}\right) \\
\dot{z}=3 \sin (x)-b z
\end{array}\right.
$$

Infinitely many attractors in the dimension of $x$ will also get doubled according to the dimension of $y$, as shown in Fig. 19. As proved, any of those reproduced coexisting attractors share the unified set of Lyapunov exponents [0.2328, 0, -0.7328]. As collected in Table. 2 , in fact, all those reproduced coexisting attractors have almost the same sets of Lyapunov exponents.
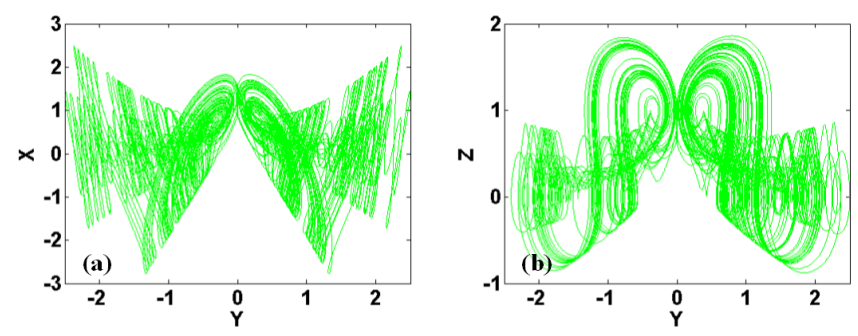

Figure 16 Pseudo-double-scroll attractor in system (7) with $a=$ $3.55, b=0.5, c=d=0$ under initial condition $(1,2,1)$ : (a) $y-x$, (b) $y-z$.
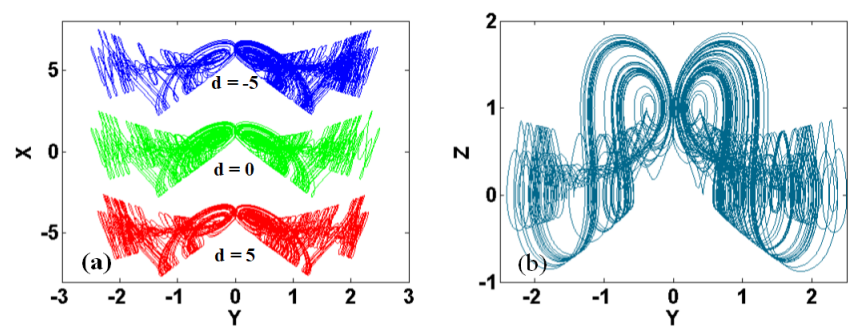

Figure 17 Chaotic attractors in system (7) with $a=3.55, b=0.5$, $c=0$ : (a) $y-x$, (b) $y$-z. Here in the left plot, red is for $d=5$ and IC $=(-4,2,1)$, green is for $d=0$ and IC $=(1,2,1)$ and blue is for $d=-5$ and $\mathrm{IC}=(6,2,1)$; because of the same plot in $y-z$ plane, here a third color is applied for representing each coexisting attractor.

\section{POLARITY CONTROL BASED ON OFFSET BOOSTING}

Moreover, the offset boosting can introduce polarity reversal leading to other regimes of systems with coexisting attractors if the polarity balance is maintained typically conditional symmetry is expectable (Li et al. 2020c). Revising the original system to be,

$$
\left\{\begin{array}{l}
\dot{x}=1-a y z \\
\dot{y}=z^{2}-|z| \\
\dot{z}=x-b z
\end{array}\right.
$$

The offset in the dimension of $x$ and $y$ win the polarity return in the right hand of the equation breeding conditional symmetry,

$$
\left\{\begin{array}{l}
\dot{x}=1-a(|y|-e) z \\
\dot{y}=z^{2}-|z| \\
\dot{z}=(|x|-f)-b z
\end{array}\right.
$$

It is clear that the offset boosting in the dimension $x$ and $y$ does not change the polarity of the left hand of Eq. (10) but gives birth to polarity reversal by the absolute value function, which is 


\begin{tabular}{llll}
\hline System & Parameters & $L E_{S}$ & $D_{K Y}$ \\
\hline$(1)$ & $a=3.55, b=0.5$ & $0.1510,0,-0.6510$ & 2.2319 \\
$(2)$ (Case A) & $a=3.55, b=0.5$ & $0.1510,0,-0.6510$ & 2.2319 \\
$(3)$ (Case B) & $a=3.55, b=0.5$ & $0.1510,0,-0.6510$ & 2.2319 \\
$(4)$ (Case C) & $a=3.55, b=0.5$ & $0.23401,0,-0.73402$ & 2.3188 \\
$(4)$ (Case D) & $a=3.55, b=0.5$ & $0.14275,0,-0.64275$ & 2.2221 \\
$(5)$ (Case E) & $a=2.2, b=0.7$ & $0.13415,0,-0.83416$ & 2.1608 \\
$(4)$ (Case F) & $a=3.57, b=0.7$ & $0.22152,0,-0.93029$ & 2.2381 \\
$(6)$ (Case F) & $a=4.5, b=0.7$ & $0.12917,0,-0.82917$ & 2.1558 \\
$(7)$ & $a=3.55, b=0.5$ & $0.1477,0,-0.64335$ & 2.2228 \\
\hline
\end{tabular}
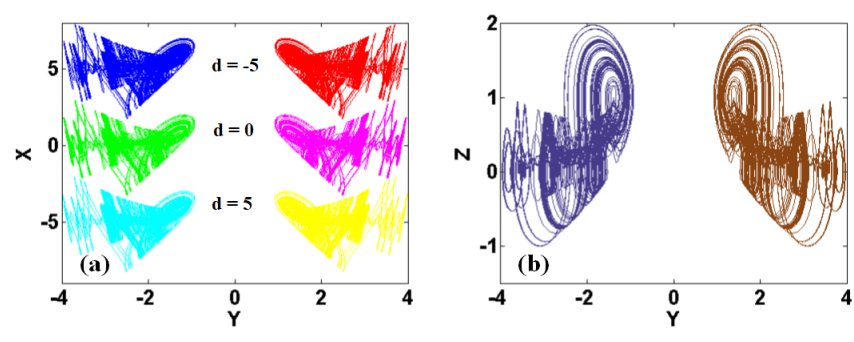

Figure 18 Chaotic attractors in system (7) with $a=3.55, b=0.5$, $c=1$ : (a) $y-x$, (b) $y$ - $z$. Here in the left plot, red is for $d=-5$ and IC $=(6,1,1)$, blue is for $d=-5$ and IC $=(6,-1,1)$, green is for $d=0$ and IC $=(1,-1,1)$, magenta is for $d=0$ and IC $=(1,1$, 1 ), yellow is for $d=5$ and IC $=(-4,1,1)$ and cyan is for $d=5$ and IC $=(-4,-1,1)$; because of the same plot in $y$ - $z$ plane, here a third color is applied for representing each coexisting attractor.
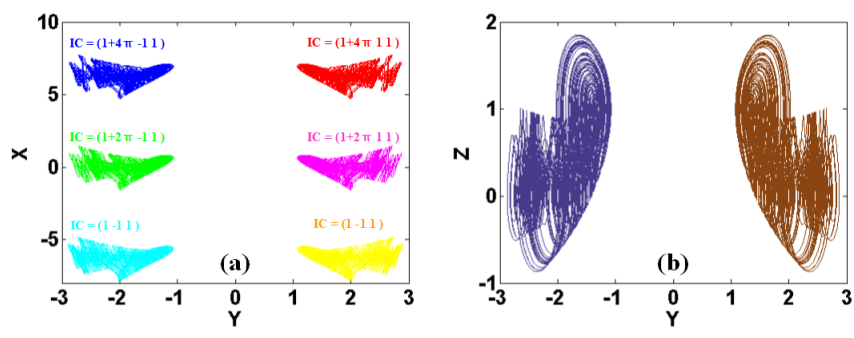

Figure 19 Chaotic attractors in system (8) with $a=3.55, b=0.5$, $c=1$ : (a) $y-x$, (b) $y$-z. Here because of the same plot in $y-z$ plane, a third color is applied for representing each coexisting attractor. counteracted by the polarity reversal of $z$ leading to conditional reflectional symmetry. As depicted in Fig. 20, coexisting chaotic attractors are produced by 2-D offset boosting in $x$ and $y$ dimensions where the polarity balance is retained by the inverse of $z$.
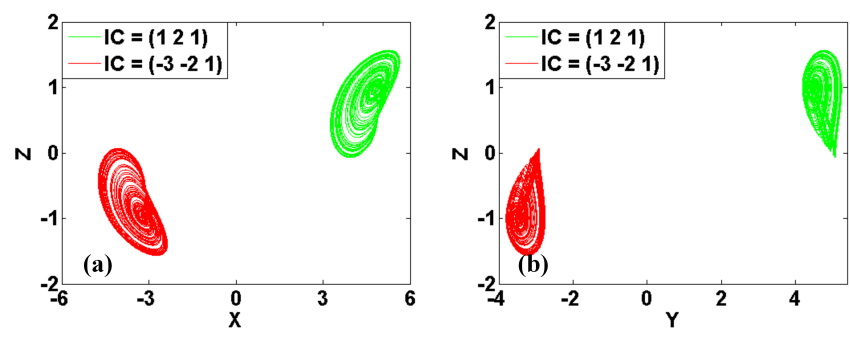

Figure 20 Coexisting attractors in conditional symmetrical system (10) with $a=2, b=0.8, e=f=4$ induced by 2-D offset boosting in $x$ and $y$ dimensions: (a) $x-z$, (b) $y-z$.

As pointed in (Li and Sprott 2017; Li et al. 2021), offset boosting may create flexibly-selected repellor if it exists in a function for equilibria controlling. With the transformation like,

$$
\left\{\begin{array}{l}
\dot{x}=(1-a(|y|-e) z) p \\
\dot{y}=\left(z^{2}-|z|\right) p \\
\dot{z}=((|x|-f)-b z) p
\end{array}\right.
$$

one of the coexisting attractors turns to be a repellor when the function $p$ is introduced as $p=y$ or $p=y-1$, as shown in Fig. 21 . Since the coexisting attractors scatter in the $y$ dimension with relatively larger distance, here the offset in the equilibria plane $y=1$ creates desired repellor. Moreover, system (9) can be revised to be a time-reversable chaotic system (Li et al. 2021),

$$
\left\{\begin{array}{l}
\dot{x}=1-a y(|z|-g) \\
\dot{y}=(|z|-g)^{2}-|| z|-g| \\
\dot{z}=x-b(|z|-g)
\end{array}\right.
$$


The time reversable property can be proved by the invariance of system (12) under the transformation of $t \rightarrow-t, x \rightarrow-x, y \rightarrow-y, z \rightarrow z+d$. Polarity balance is maintained by the offset boosting in the dimension of $z$ giving coexisting attractor and repellor as shown in Fig. 22.
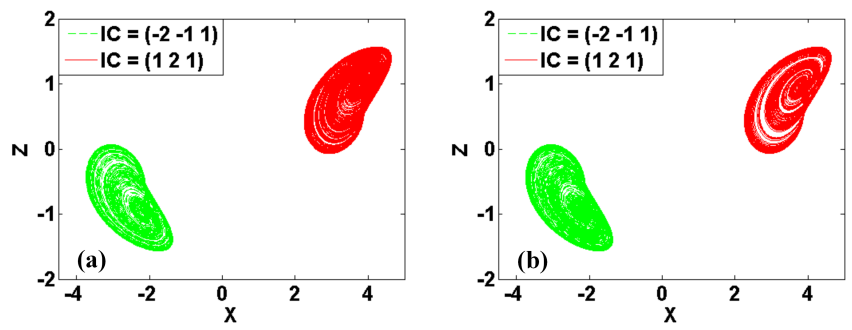

Figure 21 Coexisting chaotic attractor (red) and repellor (green) of system (11) with $a=2, b=0.8, e=f=3$ : (a) $p=y$, (b) $p=y-1$.
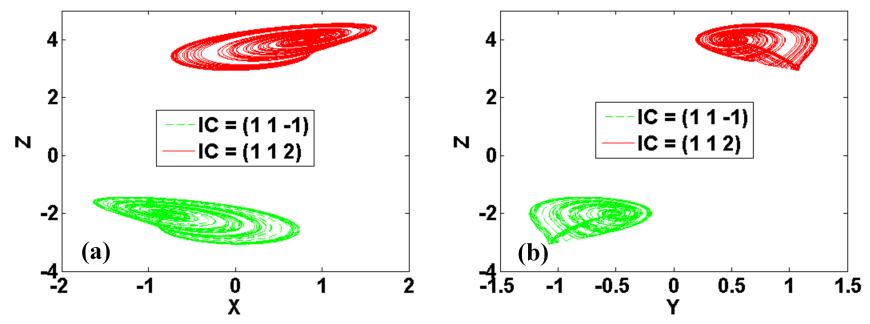

Figure 22 Coexisting chaotic attractor (red) and repellor (green) of system (12) with $a=2, b=0.8, g=3$ : (a) $x-z$, (b) $y-z$.

\section{RESULTS AND DISCUSSION}

Offset boosting of a chaotic signal or attractor represents corresponding attractor boosting. Extra introduced constant and initial condition can both trigger this process giving attractor boosting with any desired offset and producing chaotic signals with designed averaged values in a continuous or a discrete way. From the above demonstration, we can select proper approach to realize offset boosting according to our restriction. For obtaining a chaotic waveform with desired average, the offset booster is reliable since any DC power supply is easily available and selectable. For polarity control from the bipolar signal to monopolar signal or vice versa, direct constant control with a DC source can also accomplish this task effectively and output any desired stable signals.

For free access to the attractor with various offset, periodic function or absolute value function can also be introduced for attractor reproducing or doubling, where initial condition is applied to visit any included attractor. For this purpose, periodic functions may be introduced for free attractor reproducing. Attractor growing may happen in this case. Absolute value function can be pulled in a dynamical system for attractor doubling. For some specifical systems, the insert of absolute value function may bring a polarity reversal giving conditional symmetry or time-reversable conditional symmetry.

Parameter-oriented and initial-condition-oriented offset boosting can be combined together for engineering application according to the engineering restriction. Furthermore, some other piecewise linear functions (Li et al. 2020a,b) can be designed for attractor selecting and reproducing, where all the selected attractors can be arranged in any dimension or in any order if the offset is controlled harmoniously for all the attractors. Dynamic editing is heading to this direction for further exploration.

\section{Acknowledgments}

This work was supported financially by the National Natural Science Foundation of China (Grant No.: 61871230), the Natural Science Foundation of Jiangsu Province (Grant No.: BK20181410), and a project funded by the Priority Academic Program Development of Jiangsu Higher Education Institutions.

\section{Conflicts of interest}

The authors declare that there is no conflict of interest regarding the publication of this paper.

\section{LITERATURE CITED}

Akgul, A., C. Arslan, and B. Aricioglu, 2019 Image authentication using chaotic mixing systems. Chaos theory and applications 1: $1-18$.

Akgul, A., H. Calgan, I. Koyuncu, I. Pehlivan, and A. Istanbullu, 2016 Chaos-basedengineering applications with a 3D chaotic system without equilibrium points. Nonlinear Dynamics 84: 481-495.

Bao, H., W. Liu, J. Ma, and H. Wu, 2020 Memristor initial-offset boosting in memristive hr neuron model with hidden firing patterns. International Journal of Bifurcation and Chaos 30: 2030029.

C. Sprott, J., 2010 Elegant chaos: algebraically simple chaotic flows. World Scientific p. 304.

C. Sprott, J. and A. Xiong, 2015 Classifying and quantifying basins of attraction. Chaos 25: 2230.

Chen, M., X. Ren, H. Wu, Q. Xu, and B. Bao, 2019 Periodically varied initial offset boosting behaviors in a memristive system with cosine memductance. Frontiers of Information Technology and Electronic Engineering 20: 1706-1716.

Chen, M., X. Ren, H. Wu, Q. Xu, and B. Bao, 2020 Interpreting initial offset boosting via reconstitution in integral domain. Chaos, Solitons and Fractals 131: 109544.

Ding, D., X. Shan, J. Luo, Y. Hu, and L. Ding, 2020 Initial boosting phenomenon of a fractional-order hyperchaotic system based on dual memristors. Modern Physics Letters B 34: 2050191.

Falco, A. D., T. F. Krauss, and A. Fratalocchi, 2012 Lifetime statistics of quantum chaos studied by a multiscale analysis. Applied Physics Letters 100: 1914-1917.

Gu, S., S. He, H. Wang, and B. Du, 2021 Analysis of three types of initial offset-boosting behavior for a new fractional-order dynamical system. Chaos Solitons and Fractals 143: 110613.

Kengne, J., G. Leutcho, and A. Telem, 2018 Reversals of period doubling, coexisting multiple attractors, and offset boosting in a novel memristive diode bridge-based hyperjerk circuit. Analog Integrated Circuits and Signal Processing 101: 379-399.

Kingni, S. T., K. Rajagopal, S. Cicek, A. Srinivasan, and Karthikeyan, 2020 Dynamic analysis, FPGA implementation, and cryptographic application of an autonomous $5 \mathrm{~d}$ chaotic system with offset boosting. Frontiers of Information Technology and Electronic Engineering 21: 950-961.

Li, C., G. Chen, J. Kurths, T. Lei, and Z. Liu, 2020a Dynamic transport: from bifurcation to multistability. Communications in Nonlinear Science and Numerical Simulation 95: 105600.

Li, C., Z. Gu, Z. Liu, S. Jafari, and T. Kapitaniak, 2021 Constructing chaotic repellors. Chaos Solitons and Fractals 142: 110544. 
Li, C., T. Lei, X. Wang, and G. Chen, 2020b Dynamics editing based on offset boosting. Chaos 30: 063124.

Li, C., T. Lu, G. Chen, and H. Xing, 2019 Doubling the coexisting attractors. Chaos 29: 051102.

Li, C. and J. C. Sprott, 2016 Variable-boostable chaotic flows. International Journal for Light and Electron Optics 127: 10389-10398.

Li, C. and J. C. Sprott, 2017 How to bridge attractors and repellors. International Journal of Bifurcation and Chaos 27: 1750149.

Li, C., J. C. Sprott, W. Hu, and Y. Xu, 2017a Infinite multistability in a self-reproducing chaotic system. International Journal of Bifurcation and Chaos 27: 1750160.

Li, C., J. Sun, J. C. Sprott, and T. Lei, 2020c Hidden attractors with conditional symmetry. International Journal of Bifurcation and Chaos 30: 2030042.

Li, C., W. Xiong, and G. Chen, 2017b Diagnosing multistability by offset boosting. Nonlinear Dynamics 90: 1335-1341.

Liu, J., G. Chen, and X. Zhao, 2020 Generalized synchronization and parameters identification of different-dimensional chaotic systems in the complex field. Fractals 29: 2150081-1-13.

Lu, T., C. Li, S. Jafari, and F. Min, 2019 Controlling coexisting attractors of conditional symmetry. International Journal of Bifurcation and Chaos 29: 1950207.

Lu, T., C. Li, X. Wang, C. Tao, and Z. Liu, 2020 A memristive chaotic system with offset-boostable conditional symmetry. The European Physical Journal Special Topics 229: 1059-1069.

Ma, C., J. Mou, L. Xiong, S. Banerjee, and X. Han, 2021 Dynamical analysis of a new chaotic system: asymmetric multistability, offset boosting control and circuit realization. Nonlinear Dynamics 103: $1-14$

Mezatio, B. A., M. T. Motchongom, B. W. Tekam, R. Kengne, R. Tchitnga, et al., 2019 A novel memristive 6d hyperchaotic autonomous system with hidden extreme multistability. Chaos Solitons and Fractals 120: 100-115.

Wang, S., C. Wang, and C. Xu, 2020 An image encryption algorithm based on a hidden attractor chaos system and the knuth-durstenfeld algorithm. Optics and Lasers in Engineering 128: 105995.

Wu, H., Y. Ye, B. Bao, M. Chen, and Q. Xu, 2019a Memristor initial boosting behaviors in a two-memristor-based hyperchaotic system. Chaos Solitons and Fractals 121: 178-185.

Wu, H., Y. Ye, M. Chen, Q. Xu, and B. Bao, 2019b Periodically switched memristor initial boosting behaviors in memristive hypogenetic jerk system. IEEE Access 7: 1-1.

Yuan, F., Y. Deng, Y. Li, and G. Wang, 2019 The amplitude, frequency and parameter space boosting in a memristor-meminductor-based circuit. Nonlinear Dynamics 96: 389-405.

Zhang, S., Y. Zeng, Z. Li, and C. Zhou, 2018 Hidden extreme multistability, antimonotonicity and offset boosting control in a novel fractional-order hyperchaotic system without equilibrium. International Journal of Bifurcation and Chaos 28: 1850167.

Zhang, S., J. Zheng, X. Wang, Z. Zeng, and S. He, 2020 Initial offset boosting coexisting attractors in memristive multi-double-scroll hopfield neural network. Nonlinear Dynamics 102: 2821-2841.

Zhao, X., J. Liu, J. Mou, C. Ma, and F. Yang, 2020 Characteristics of a laser system in complex field and its complex selfsynchronization. The European Physical Journal Plus 135: 1-17.

How to cite this article: $\mathrm{Li}, \mathrm{C} .$, Jiang, Y., and Ma, X. On offset boosting in chaotic system. Chaos Theory and Applications, 3(2), 47-54, 2021. 